SU-4240-652

KEK preprint 96-150

hep-ph/9612358

December, 1996

\title{
The Pion Decay Constant and the $\rho$-Meson Mass at Finite Temperature in the Hidden Local Symmetry
}

\author{
Masayasu HARADA \\ Department of Physics, Syracuse University, \\ Syracuse, NY 13244-1130, USA \\ Akihiro ShiBatai \\ National Laboratory for High Enegr Physics (KEK), \\ Tsukuba 305, Japan
}

\begin{abstract}
We study the temperature dependence of the pion decay constant and $\rho$-meson mass in the hidden local symmetry model at one loop. Using the standard imaginary time formalism, we include the thermal effect of $\rho$ meson as well as that of pion. We show that the pion gives a dominant contribution to the pion decay constant and $\rho$-meson contribution slightly decreases the critical temperature. The $\rho$-meson pole mass increases as $T^{4} / m_{\rho}^{2}$ at low temperature dominated by the pion-loop effect. At high temperature, although the pion-loop effect decreases the $\rho$-meson mass, the $\rho$-loop contribution overcomes the pion-loop contribution and $\rho$-meson mass increases with temperature. We also show that the conventional parameter $a$ is stable as the temperature increases.
\end{abstract}

\footnotetext{
* e-mail address : mharada@npac.syr.edu

${ }^{\dagger}$ e-mail address : ashibata@mail.kek.jp
} 


\section{INTRODUCTION}

One of the most remarkable feature of QCD in the low energy is that the approximate chiral symmetry is spontaneously broken, and the approximate Nambu-Goldstone (NG) boson appears. The pion is regarded as the NG boson and the resultant low energy theorems successfully reproduce the low energy physics of pions. In the hot and/or dense matter, however, the quark condensate melts at some critical point, and the chiral symmetry is restored. This chiral phase transition was widely studied (for recent reviews, see e.g., Refs. [1 [3]).

Several experiments such as RHIC are planned to measure the effects in hot and/or dense matter. One of the interesting quantities in the hot and/or dense matter is the change of $\rho$ meson mass. In Refs. [2, [4] it was proposed that the $\rho$-meson mass scaled like the pion decay constant in the hot and/or dense matter, and vanished at chiral phase transition point. The low temperature theorem for $\rho$-meson was obtained by using the current algebra [5], which showed that the $\rho$-meson mass was stable in the low temperature region. The thermal pion effect to $\rho$-meson mass was studied by using the effective theories [6], and it was shown that the $\rho$ meson mass slightly increased with the temperature. On the other hand, the thermal effect of nucleon was shown to give a negative contribution. [7] However, the thermal effects of $\rho$-meson itself was not included in these analysis. In Ref. [8] the thermal effects of heavier mesons to the quark condensate were included into the chiral perturbation analysis by using the dilute gas approximation. The $\rho$ meson seems to give a non-negligible effect near critical temperature. Then, it is interesting to see the thermal effect of $\rho$ meson, especially to the $\rho$-meson mass, by including it systematically.

To include the thermal $\rho$-meson effect systematically it is convenient to use the effective Lagrangian of the pion and the $\rho$-meson. There are several models which include pion and $\rho$ meson, among which we study the hidden local symmetry model. [9] For the parameter choice $a=2$ this model successfully predicts the following phenomenological facts [10]: the $\rho$-coupling universality, $g_{\rho \pi \pi}=g$ [11]; the $\mathrm{KSRF}$ relation (II), $m_{\rho}^{2}=2 g_{\rho \pi \pi}^{2} f_{\pi}^{2}$ [12]; the $\rho$ meson dominance of the electromagnetic form factor of the pion, $g_{\gamma \pi \pi}=0$ [11]. Moreover, we obtain the $\operatorname{KSRF}(\mathrm{I})$ relation, $g_{\rho}=2 f_{\pi}^{2} g_{\rho \pi \pi}$ [12] as a "low energy theorem" of hidden local symmetry [13], which was first proven at tree level [14] and then at any loop order [15]. One loop corrections to the above predictions were studied in the Landau gauge [16]. The systematic loop expansion like chiral perturbation was studied in Ref. [17], where the expansion was done by regarding the $\rho$-meson as a light particle.

In this paper we study the temperature dependence of the pion decay constant and the $\rho$-meson mass by using the hidden local symmetry model at one loop. It is also interesting to see the temperature dependence of the parameter $a$, which is related to the above successful phenomenological predictions. One-loop calculation will be done by using the standard imaginary time formalism. [18] The renormalization is done in the low energy limit as shown 
in Ref. [16].

This paper is organized as follows. In section [1], we briefly review the hidden local symmetry, and introduce an $R_{\xi}$-like gauge-fixing term and the corresponding ghost Lagrangian. In section III, we show the temperature dependence of the pion decay constant at finite temperature. The temperature dependence of the parameter $a$ is also studied. Section IV is devoted to study the temperature dependence of the $\rho$-meson mass by using the on-shell-like renormalization condition. Finally, the summary and discussion are given in section $\mathrm{V}$. To avoid complexity, the polarization tensors at finite temperature and complicated functions are summarized in Appendices $\mathrm{A}$ and B, respectively. We also study the $\rho$-meson propagator at finite temperature in Appendix C.

\section{HIDDEN LOCAL SYMMETRY}

Let us start with the $\left[\mathrm{SU}(N)_{\mathrm{L}} \times \mathrm{SU}(N)_{\mathrm{R}}\right]_{\text {global }} \times\left[\mathrm{SU}(N)_{\mathrm{V}}\right]_{\text {local }}$ "linear" model. [10 We introduce two $\mathrm{SU}(N)$-matrix valued variables, $\xi_{\mathrm{L}}(x)$ and $\xi_{\mathrm{R}}(x)$, which transform as

$$
\xi_{\mathrm{L}, \mathrm{R}}(x) \rightarrow \xi_{\mathrm{L}, \mathrm{R}}^{\prime}(x)=h(x) \xi_{\mathrm{L}, \mathrm{R}}(x) g_{\mathrm{R}, \mathrm{L}}^{\dagger}
$$

where $h(x) \in\left[\mathrm{SU}(N)_{\mathrm{V}}\right]_{\text {local }}$ and $g_{\mathrm{L}, \mathrm{R}} \in\left[\mathrm{SU}(N)_{\mathrm{L}, \mathrm{R}}\right]_{\text {global }}$. These variables are parameterized as

$$
\xi_{\mathrm{L}, \mathrm{R}}(x) \equiv e^{i \sigma(x) / f_{\sigma}} e^{\mp i \pi(x) / f_{\pi}}, \quad\left[\pi(x) \equiv \pi^{a}(x) T_{a}, \sigma(x) \equiv \sigma^{a}(x) T_{a}\right]
$$

where $\pi$ and $\sigma$ are the pion and the "compensator" (would-be Nambu-Goldstone field) to be "absorbed" into the hidden gauge boson (the $\rho$ meson), respectively, and $f_{\pi}$ and $f_{\sigma}$ are the corresponding decay constants in the chiral symmetric limit. The covariant derivatives are defined by

$$
\begin{aligned}
D_{\mu} \xi_{\mathrm{L}} & \equiv \partial_{\mu} \xi_{\mathrm{L}}-i g V_{\mu} \xi_{\mathrm{L}}+i \xi_{\mathrm{L}} \mathcal{L}_{\mu} \\
D_{\mu} \xi_{\mathrm{R}} & \equiv \partial_{\mu} \xi_{\mathrm{R}}-i g V_{\mu} \xi_{\mathrm{R}}+i \xi_{\mathrm{R}} \mathcal{R}_{\mu}
\end{aligned}
$$

where $g$ is the gauge coupling constant of the hidden local symmetry, $V_{\mu}\left(\equiv V_{\mu}^{a} T_{a}\right)$ the hidden gauge boson field (the $\rho$ meson), and $\mathcal{L}_{\mu}$ and $\mathcal{R}_{\mu}$ denote the external fields gauging the $\left[\mathrm{SU}(N)_{\mathrm{L}}\right]_{\text {global }}$ and $\left[\mathrm{SU}(N)_{\mathrm{R}}\right]_{\text {global }}$, respectively. The Lagrangian of $\left[\mathrm{SU}(N)_{\mathrm{L}} \times \mathrm{SU}(N)_{\mathrm{R}}\right]_{\text {global }}$ $\times\left[\mathrm{SU}(N)_{\mathrm{V}}\right]_{\text {local }}$ "linear" model is given by 9,10 ,

\footnotetext{
‡The massive Yang-Mills approach [19] gives Lagrangian of the same form if we take unitary gauge of hidden local symmetry. This is equivalent to the hidden local gauge method at tree level.
} 


$$
\mathcal{L}=f_{\pi}^{2} \operatorname{tr}\left[\left(\hat{\alpha}_{\mu \perp}\right)^{2}\right]+a f_{\pi}^{2} \operatorname{tr}\left[\left(\hat{\alpha}_{\mu \|}\right)^{2}\right]-\frac{1}{2} \operatorname{tr}\left[V_{\mu \nu} V^{\mu \nu}\right]
$$

where $a$ is a constant, and $\hat{\alpha}_{\mu \perp}$ and $\hat{\alpha}_{\mu \|}$ are the covariantized Maurer-Cartan one-forms [14]:

$$
\begin{aligned}
\hat{\alpha}_{\mu \perp} & \equiv \frac{D_{\mu} \xi_{\mathrm{L}} \cdot \xi_{\mathrm{L}}^{\dagger}-D_{\mu} \xi_{\mathrm{R}} \cdot \xi_{\mathrm{R}}^{\dagger}}{2 i} \\
\hat{\alpha}_{\mu \|} & \equiv \frac{D_{\mu} \xi_{\mathrm{L}} \cdot \xi_{\mathrm{L}}^{\dagger}+D_{\mu} \xi_{\mathrm{R}} \cdot \xi_{\mathrm{R}}^{\dagger}}{2 i}
\end{aligned}
$$

Normalizing the kinetic term of $\sigma$, we find [20]

$$
f_{\sigma}^{2}=a f_{\pi}^{2}
$$

For the parameter choice $a=2$ at tree level, this model predicts the following phenomenological facts [10]:

1. $g_{\rho \pi \pi}=g$ (universality of the $\rho$-coupling) [11;

2. $m_{\rho}^{2}=2 g_{\rho \pi \pi}^{2} f_{\pi}^{2}(\mathrm{KSRF} \mathrm{II})$ 12];

3. $g_{\gamma \pi \pi}=0$ ( $\rho$-meson dominance of the electromagnetic form factor of the pion) [11].

Moreover, independently of the parameter $a$, this model predicts the KSRF relation [12 (version I)

$$
g_{\rho}=2 f_{\pi}^{2} g_{\rho \pi \pi}
$$

as a "low energy theorem" of hidden local symmetry [13], which was first proven at tree level [14 and then at any loop order [15].

In this paper, to consider the loop effects of hidden local symmetry, we introduce an $R_{\xi}$-gauge-like [21] gauge-fixing and a Faddeev-Popov ghost Lagrangian corresponding to the hidden local gauge boson. These are given by [16]

$$
\begin{aligned}
\mathcal{L}_{\mathrm{GF}+\mathrm{FP}}= & -\frac{1}{\alpha} \operatorname{tr}\left[\left(\partial^{\mu} V_{\mu}\right)\right]+\frac{i}{2} a g f_{\pi}^{2} \operatorname{tr}\left[\partial^{\mu} V_{\mu}\left(\xi_{\mathrm{L}}-\xi_{\mathrm{L}}^{\dagger}+\xi_{\mathrm{R}}-\xi_{\mathrm{R}}^{\dagger}\right)\right] \\
& +\frac{1}{16} \alpha a^{2} g^{2} f_{\pi}^{4}\left\{\operatorname{tr}\left[\left(\xi_{\mathrm{L}}-\xi_{\mathrm{L}}^{\dagger}+\xi_{\mathrm{R}}-\xi_{\mathrm{R}}^{\dagger}\right)^{2}\right]-\frac{1}{N}\left(\operatorname{tr}\left[\xi_{\mathrm{L}}-\xi_{\mathrm{L}}^{\dagger}+\xi_{\mathrm{R}}-\xi_{\mathrm{R}}^{\dagger}\right]\right)^{2}\right\} \\
& +i \operatorname{tr}\left[\bar{C}\left\{2 \partial^{\mu} D_{\mu} C+\frac{1}{2} \alpha a g^{2} f_{\pi}^{2}\left(C \xi_{\mathrm{L}}+\xi_{\mathrm{L}}^{\dagger} C+C \xi_{\mathrm{R}}+\xi_{\mathrm{R}}^{\dagger} C\right)\right\}\right]
\end{aligned}
$$

where $\alpha$ denotes a gauge parameter and $C$ denotes a ghost field. In this paper we choose the Landau gauge, $\alpha=0$. In this gauge the would-be Nambu-Goldstone (NG) boson $\sigma$ is still massless, no other vector-scalar interactions are created and the ghost field couples only to the hidden local gauge field. 
The renormalization is done by introducing counter terms. [16,17] Here following Ref. [16], we use the $Z$ factors defined by

$$
\begin{array}{ll}
g_{0}=Z_{g} g, & V_{0 \mu}=Z_{V}^{1 / 2} V_{\mu} \\
\pi_{0}=Z_{\pi}^{1 / 2} \pi, & \sigma_{0}=Z_{\sigma}^{1 / 2} \sigma \\
f_{\pi 0}=Z_{\pi}^{1 / 2} f_{\pi}, & f_{\sigma 0}=Z_{\sigma}^{1 / 2} f_{\sigma} .
\end{array}
$$

We renormalize the theory at zero temperature, then the corrections from the thermal pion, $\rho$ meson, and so on are calculated to be finite.

\section{THE TEMPERATURE DEPENDENCE OF DECAY CONSTANTS}

In this section we study the temperature dependences of the decay constants of pion and sigma. We also study the temperature dependence of the parameter $a$, which is related to the successful phenomenological predictions of hidden local symmetry.

To define the pion decay constant at finite temperature, we consider a Green function for the axial-vector current $A_{a}^{\mu}$

$$
\delta_{a b} G_{\mathcal{A}}^{\mu \nu}\left(p_{0}, \vec{p} ; T\right) \equiv \text { F.T. }\left\langle 0\left|A_{a}^{\mu} A_{b}^{\nu}\right| 0\right\rangle_{T}
$$

Since the axial-vector current is conserved, we can decompose this Green function into longitudinal and transverse pieces:

$$
G_{\mathcal{A}}^{\mu \nu}\left(p_{0}, \vec{p} ; T\right)=P_{T}^{\mu \nu} G_{\mathcal{A} T}+P_{L}^{\mu \nu} G_{\mathcal{A} L}
$$

where polarization tensors $P_{L}$ and $P_{T}$ are defined in Eq. (A1). It is natural to define the pion decay constant at finite temperature through the longitudinal component in the low energy limit'w: [22]

$$
f_{\pi}^{2}(T) \equiv-\lim _{p_{0} \rightarrow 0} G_{\mathcal{A L}}\left(p_{0}, \vec{p}=0\right)
$$

There are two types of contributions to this Green function in our model: (i) the pion exchange diagrams, and (ii) the contact or one-particle irreducible (1PI) diagrams. The

\footnotetext{
$\S$ To perform the complete renormalization at one loop, we need higher order counter terms. [17] However, for the quantities we are studying in this paper, the counter terms induced from $Z$-factors in Eq. (9) are enough to renormalize the divergences. Then we do not explicitly introduce such higher order counter terms here.

**Even when we use the transverse part instead of the longitudinal part to define $f_{\pi}(T)$ in Eq. (12), we obtain the same result: $G_{\mathcal{A} T}\left(p_{0}, \vec{p}=0\right)=G_{\mathcal{A} L}\left(p_{0}, \vec{p}=0\right)$.
} 
contribution (i) is proportional to $p_{\mu}$ or $p_{\nu}$ at one loop. At most only one of the $A_{a}^{\mu}$ - $\pi$ coupling can be corrected at one loop, which is not generally proportional to four momentum $p_{\mu}$. The other coupling is the tree-level one and proportional to $p_{\mu}$. When we act with the projection operator $P_{L \mu \nu}$, the term proportional to $p_{\mu}$ vanishes. Due to the current conservation we have same kinds of contributions from 1PI diagrams: those are roughly proportional to $g_{\mu \nu}$ instead of $p_{\mu}$. Then we will calculate only the 1PI diagrams.

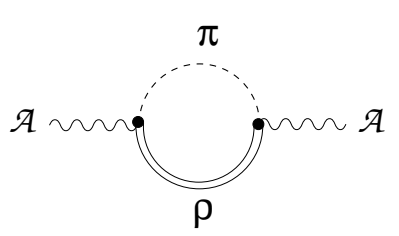

(a)

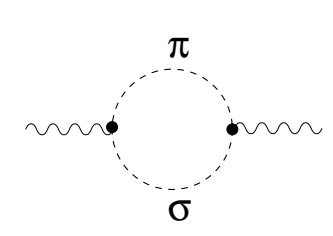

(b)

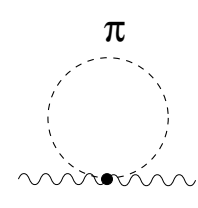

(c)

FIG. 1. Feynman diagrams contributing to the one particle irreducible part of the axial-vector current two-point function.

There exist three 1PI diagrams which contribute to $G_{\mathcal{A}}^{\mu \nu}$ at one-loop level: (a) $\pi+\rho$ loop, (b) $\pi+\sigma$ loop, (c) $\pi$ tad-pole, which are shown in Fig. 1. These diagrams include ultraviolet divergences, which are renormalized by $Z_{\pi}$ in Eq. (9). By taking a suitable subtraction scheme at zero temperature, all the divergences including finite corrections in the low energy limit are absorbed into $Z_{\pi}$. 15, 16 Then the loop diagrams generate only the temperature dependent part. By using standard imaginary time formalism [18] we obtain

$$
\begin{aligned}
& G_{\mathcal{A} L}^{(a)}\left(p_{0}, \vec{p}=0\right)=\frac{N}{2} \frac{a}{\pi^{2}}\left[\frac{5}{6} I_{2}-J_{1}^{2}+\frac{1}{3 m_{\rho}^{2}}\left(I_{4}-J_{1}^{4}\right)\right] \\
& G_{\mathcal{A} L}^{(b)}\left(p_{0}, \vec{p}=0\right)=\frac{N}{2} \frac{a}{6 \pi^{2}} I_{2} \\
& G_{\mathcal{A} L}^{(c)}\left(p_{0}, \vec{p}=0\right)=\frac{N}{2} \frac{1-a}{\pi^{2}} I_{2}
\end{aligned}
$$

where the functions $I_{n}$ and $J_{m}^{n}$ are defined in appendix B. If we saw diagrams naively, we might think the diagram (c) gives the dominant contribution. However, each diagram does generate the dominant contribution $I_{2}=\left(\pi^{2} / 6\right) T^{2}$. Nevertheless, we see from Eq. (13) that the terms proportional to $a I_{2}$ are cancelled among three diagrams. The total contribution is given by

$$
f_{\pi}^{2}(T)=f_{\pi}^{2}-\frac{N}{2} \frac{1}{\pi^{2}}\left[I_{2}-a J_{1}^{2}+\frac{a}{3 m_{\rho}^{2}}\left(I_{4}-J_{1}^{4}\right)\right] .
$$

When we take $m_{\rho} \rightarrow \infty$ limit in the above expression, only the $I_{2}$-term remains;

$$
\left[f_{\pi}^{\mathrm{ChPT}}(T)\right]^{2}=f_{\pi}^{2}-\frac{N}{2} \frac{I_{2}}{\pi^{2}}=f_{\pi}^{2}-\frac{N}{12} T^{2}
$$


which is consistent with the result given by Gasser-Leutwyler. 23]

We show the temperature dependence of $f_{\pi}(T)$ by a solid line in Fig. 2, where we use $f_{\pi}=93 \mathrm{MeV}, m_{\rho}=770 \mathrm{MeV}, a=2$ and $N=2$. (We use same values for numerical analysis below.) The chiral perturbation prediction Eq.(15) is shown by a dotted line in Fig. 2. Figure 2 shows that the pion loop gives a dominant contribution and $\rho$-meson loop generates a small correction. The situation is similar to the quark condensate in the chiral perturbation analysis. [8]

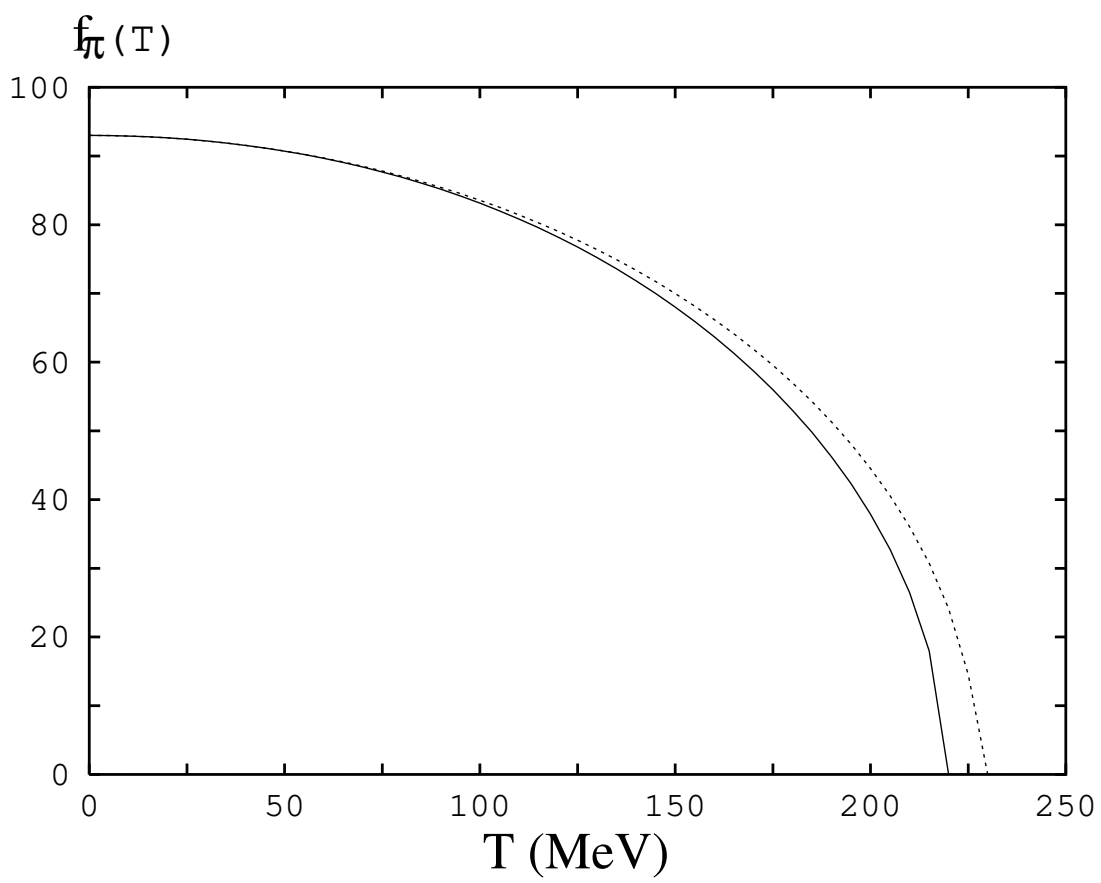

FIG. 2. The temperature dependence of the pion decay constant. The solid line denotes the total contribution, $f_{\pi}(T)$ in Eq. (14) and the dotted line denotes the $m_{\rho} \rightarrow \infty$ limit (Eq. (15)).

We should notice that the term proportional to $I_{4}$ corresponds to a part of two-loop order effects in the ordinary chiral perturbation theory. The two-loop order effects are divided into two types of diagrams: one is two-loop diagram which includes $O\left(p^{2}\right)$ vertices only and the other is one-loop diagram which includes only one $O\left(p^{4}\right)$ vertex. In chiral perturbation theory the $O\left(p^{4}\right)$ vertices are parameterized by $\ell_{1}$ and $\ell_{2}$ [24], which are saturated by the effect of $\rho$ meson. [25] Then the $I_{4}$ term generated by $\rho$ meson may be a good approximation to $O\left(p^{4}\right)$ contribution in the chiral perturbation theory.

Next, we study the temperature dependence of $f_{\sigma}$. Similar to the case of $f_{\pi}$, we start with a Green function for the vector current:

$$
\delta_{a b} G_{\mathcal{V}}^{\mu \nu}\left(p_{0}, \vec{p} ; T\right) \equiv \mathrm{F} . \mathrm{T} .\left\langle 0\left|\mathcal{V}_{a}^{\mu} \mathcal{V}_{b}^{\nu}\right| 0\right\rangle_{T}
$$

There are three types of contributions: (i) 1PI diagrams; (ii) the $\sigma$-exchange diagrams; (iii) the $\rho$-exchange diagrams. We have no strict definition of $f_{\sigma}(T)$ like $f_{\pi}(T)$ in Eq. (12), since 
$\sigma$ is not a physical particle. The $\sigma$ is a would-be Nambu-Goldstone boson which is absorbed into $\rho$ meson. In the Landau gauge, the $\rho$-exchange contribution is separately conserved, as we can show easily by using the $\rho$ propagator (C10). From the conservation of whole current, the sum of $1 \mathrm{PI}$ and $\sigma$-exchange diagrams are conserved. Then, it is reasonable to define $f_{\sigma}(T)$ like Eq. (12) through only the 1PI and $\sigma$-exchange diagrams:

$$
f_{\sigma}^{2}(T) \equiv-\lim _{p_{0} \rightarrow 0} G_{\mathcal{V} L}^{(1 \mathrm{PI}+\sigma)}\left(p_{0}, \vec{p}=0 ; T\right) .
$$

As discussed below Eq. (12) for $f_{\pi}(T)$, it is enough to calculate the 1PI diagrams to determine the temperature dependence of $f_{\sigma}(T)$.

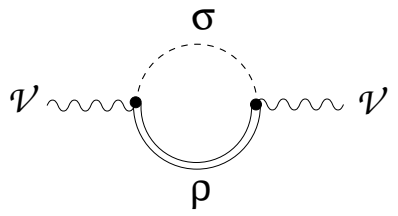

(a)

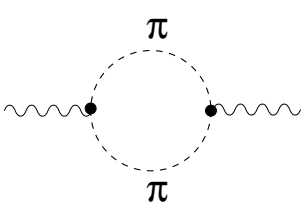

(b)

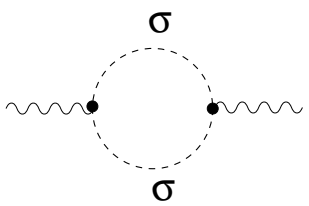

(c)

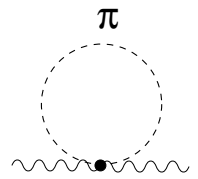

(d)

FIG. 3. Feynman diagrams for the one particle irreducible part of the vector current two-point function.

There are four diagrams which contribute to the 1PI part of $G_{\mathcal{V}}^{\mu \nu}$ at one loop, which are shown in Fig. 3. We note that we do not have $\sigma$ tad-pole contribution, since there is no $\sigma-\sigma-\mathcal{V}-\mathcal{V}$ type vertex at tree level. These four diagrams lead to the temperature dependence given by

$$
f_{\sigma}^{2}(T)=f_{\sigma}^{2}-\frac{N}{2} \frac{1}{\pi^{2}}\left[\frac{a^{2}+8 a+3}{12} I_{2}-J_{1}^{2}+\frac{1}{3 m_{\rho}^{2}}\left(I_{4}-J_{1}^{4}\right)\right] .
$$

The parameter $a$ at finite temperature is given by $a(T)=f_{\sigma}^{2}(T) / f_{\pi}^{2}(T)$ (see Eq. (6)). Using the temperature dependence of $f_{\pi}$ and $f_{\sigma}$ given in Eqs. (14) and (18), we obtain the temperature dependence of the parameter $a$ :

\footnotetext{
${ }^{\dagger \dagger}$ At tree level, the $\rho-\gamma$ mixing is proportional to $g_{\mu \nu}$. One-loop effects at finite temperature violate
} this structure, and generally the $\rho-\gamma$ mixing is decomposed into four independent polarizations: $\Pi_{\rho \mathcal{V}}^{\mu \nu}=P_{T}^{\mu \nu} \Pi_{\rho \mathcal{V} T}+P_{L}^{\mu \nu} \Pi_{\rho \mathcal{V} L}+P_{C}^{\mu \nu} \Pi_{\rho \mathcal{V} C}+P_{D}^{\mu \nu} \Pi_{\rho \mathcal{V} D}$, where the polarization tensors are given in Eq. (A1). In the low momentum limit we can easily show that $\Pi_{\rho \mathcal{V} C}$ vanishes. Since $P_{D \mu \alpha} P_{L}^{\alpha \nu}=$ $P_{D \mu \alpha} P_{T}^{\alpha \nu}=P_{L \mu \alpha} P_{T}^{\alpha \nu}=0, P_{T \mu \alpha} P_{T}^{\alpha \nu}=-P_{T \mu}^{\nu}$ and $P_{L \mu \alpha} P_{L}^{\alpha \nu}=-P_{L \mu}^{\nu}$, the $\rho$ meson exchange diagrams generate only the terms proportional to $P_{L \mu \nu}$ and $P_{T \mu \nu}$ to the vector-current two-point function. These polarization tensors vanish if they are multiplied by $p^{\mu}$. This implies that the contribution from the $\rho$-meson exchange diagrams vanishes if it is multiplied by $p^{\mu}$, and then both the $\rho$-meson exchange diagrams and $\sigma$-exchange plus 1PI diagrams conserve separately. 


$$
\frac{a(T)}{a(0)}=1+\frac{N}{2} \frac{1}{\pi^{2} f_{\pi}^{2}}\left[-\frac{(a-1)(a-3)}{12 a} I_{2}+\frac{1-a^{2}}{a} J_{1}^{2}-\frac{1-a^{2}}{3 a m_{\rho}^{2}}\left(I_{4}-J_{1}^{4}\right)\right]
$$

We show the temperature dependence of the parameter $a$ in Fig. 田, where we take $a(0)=2$. The parameter $a$ does not change very much against the temperature, i.e., $a(T) \simeq 2$ for wide range of the temperature. It was shown [16] that at zero temperature one-loop effects did not generate the direct $\gamma \pi \pi$ vertex if and only if we took a parameter choice $a=2$. The fact that $a(T) \simeq 2$ for wide range may suggest that the successful phenomenological predictions of hidden local symmetry discussed in section II hold at finite temperature in good approximation. We should note that if we take $a=1$ from the beginning the parameter $a$ does not change with temperature. Together with the fact that the parameter $a$ is not renormalized for the parameter choice $a=1$, this implies that in the "vector limit" 26] the thermal effects of the $\rho$ meson do not induce deviation from $a=1$.

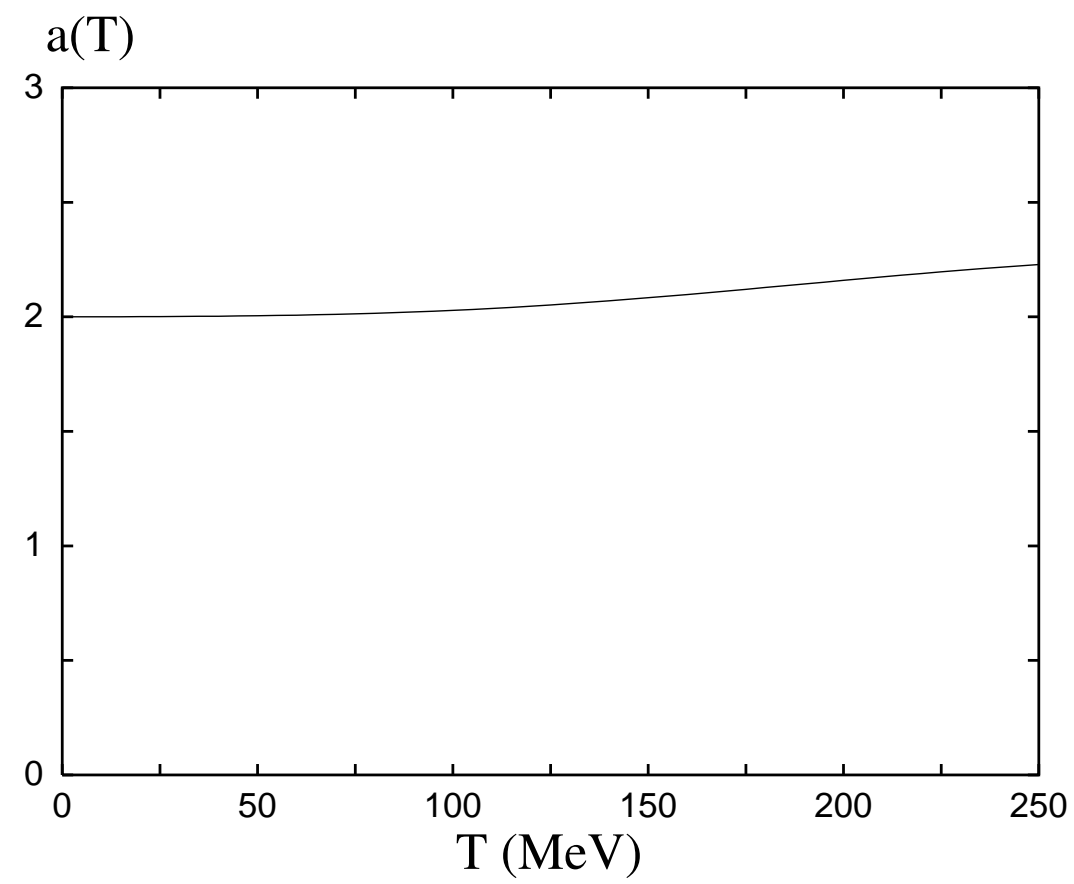

FIG. 4. The temperature dependence of the parameter $a$.

\section{THE TEMPERATURE DEPENDENCE OF $\rho$ MESON MASS}

In this section we study the temperature dependence of $\rho$-meson mass. As we show in Appendix Q, the $\rho$ and $\sigma$ propagators are separated with each other in the Landau gauge, and the $\rho$ propagator takes easy form

\footnotetext{
${ }^{\ddagger}$ Shiomi and Hatsuda [7] used similar form, where they started from the Steukelberg formalism.
} 


$$
D_{\mu \nu}=-\frac{P_{T \mu \nu}}{p^{2}-m_{\rho}^{2}+\Pi_{T}}-\frac{P_{L \mu \nu}}{p^{2}-m_{\rho}^{2}+\Pi_{L}} .
$$

It is reasonable to define the $\rho$-meson mass by using the longitudinal part.3

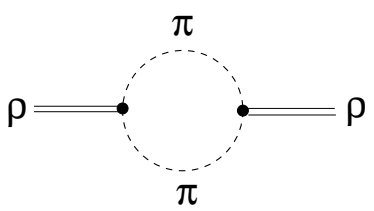

(a)

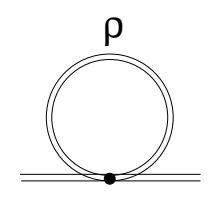

(d)

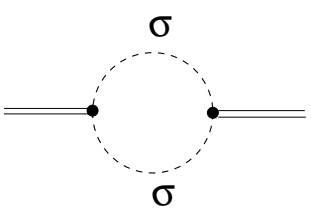

(b)

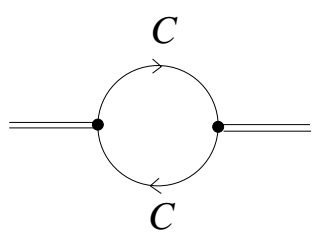

(e)

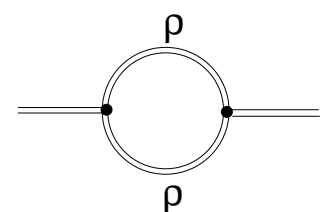

(c)

FIG. 5. Feynman diagrams contributing to the $\rho$-meson self-energy: a) $\pi$ loop, b) $\sigma$ loop, c) $\rho$ loop, d) $\rho$ tad-pole and e) ghost loop.

The one-loop diagrams contributing $\rho$ meson self-energy are shown in Fig. 5. In Ref. 16], the finite term of $Z_{V} Z_{g}^{2}$ was determined but each finite term of $Z_{V}$ and $Z_{g}$ was not determined separately. We introduce the parameter $z$ for expressing this finite term as

$$
\begin{aligned}
& Z_{V}-1=\frac{g^{2}}{(4 \pi)^{2}}\left[\frac{51-a^{2}}{12}\left\{\frac{1}{\bar{\epsilon}}-\ln m_{\rho}^{2}+\frac{5}{6}\right\}+z\right], \\
& Z_{g}-1=\frac{g^{2}}{(4 \pi)^{2}}\left[-\frac{87-a^{2}}{24}\left\{\frac{1}{\bar{\epsilon}}-\ln m_{\rho}^{2}+\frac{5}{6}\right\}-\frac{z}{2}\right] .
\end{aligned}
$$

Adding all the contributions we obtain

$$
\begin{aligned}
& \Pi_{\rho L}(p ; T=0)= \\
& \frac{N}{2} \frac{g^{2}}{(4 \pi)^{2}} m_{\rho}^{2}\left[z \delta-\frac{37}{4}-\frac{1}{6 \delta}-\frac{71}{24} \delta+\frac{11 a^{2}}{72} \delta+\frac{\delta\left(1-\delta^{2}\right)}{12} \ln (-\delta)-\frac{a^{2}}{12} \delta \ln (-\delta)\right. \\
& \left.\quad+\frac{(4-\delta)^{2}\left(12+20 \delta+\delta^{2}\right)}{6 \sqrt{4-\delta} \sqrt{\delta}} \tan ^{-1} \sqrt{\frac{\delta}{4-\delta}}-\frac{(1-\delta)^{3}\left(1+10 \delta+\delta^{2}\right)}{6 \delta^{2}} \ln (1-\delta)\right],
\end{aligned}
$$

where $\delta$ is defined by $\delta \equiv p^{2} / m_{\rho}^{2}$. By requiring that $m_{\rho}$ becomes pole mass:

$\S \S$ It should be noticed that the transverse polarization agrees with the longitudinal one in the low momentum limit: $\Pi_{\rho T}\left(p_{0}, \vec{p}=0 ; T\right)=\Pi_{\rho L}\left(p_{0}, \vec{p}=0 ; T\right)$. 


$$
\operatorname{Re} \Pi_{\rho L}\left(p^{2}=m_{\rho}^{2} ; T=0\right)=0
$$

this finite part $z$ is determined as

$$
z=\frac{99}{8}-\frac{11}{72} a^{2}-\frac{11 \sqrt{3} \pi}{4}
$$

The temperature dependence of the self-energy of $\rho$ meson obtained from the thermal pion and $\rho$ meson is calculated by using the standard imaginary time formalism. [18] The temperature dependent parts, $\Delta \Pi\left(p_{0}, \vec{p} ; T\right) \equiv \Pi\left(p_{0}, \vec{p} ; T\right)-\Pi\left(p_{0}, \vec{p} ; T=0\right)$, are given by

$$
\begin{aligned}
& \operatorname{Re} \Delta \Pi_{\rho L}^{(a)}\left(p_{0}, \vec{p}=0 ; T\right)=\frac{N}{2} \frac{g^{2}}{\pi^{2}} \frac{a^{2}}{12} G_{2}, \\
& \operatorname{Re} \Delta \Pi_{\rho L}^{(b)}\left(p_{0}, \vec{p}=0 ; T\right)=\frac{N}{2} \frac{g^{2}}{\pi^{2}} \frac{1}{12} G_{2} \\
& \operatorname{Re} \Delta \Pi_{\rho L}^{(c)}\left(p_{0}, \vec{p}=0 ; T\right)= \\
& \frac{N}{2} \frac{g^{2}}{\pi^{2}}\left[\frac{-\left(4 m_{\rho}^{2}-p_{0}^{2}\right)\left(m_{\rho}^{2}+p_{0}^{2}\right)}{4 m_{\rho}^{2}} F_{3}^{2}+\frac{-4 m_{\rho}^{4}+9 m_{\rho}^{2} p_{0}^{2}+p_{0}^{4}}{12 m_{\rho}^{4}} F_{3}^{4}-\frac{1}{3 m_{\rho}^{2}} F_{3}^{6}\right. \\
& \quad+\frac{\left(m_{\rho}^{2}+p_{0}^{2}\right)\left(m_{\rho}^{2}-p_{0}^{2}\right)^{2}}{m_{\rho}^{2}} H_{1}^{2}+\frac{\left(m_{\rho}^{2}+p_{0}^{2}\right)\left(m_{\rho}^{2}-p_{0}^{2}\right)^{2}}{3 m_{\rho}^{4}} H_{1}^{4} \\
& \left.\quad+\frac{p_{0}^{2}}{3 m_{\rho}^{2}}\left\{\frac{-\left(m_{\rho}^{2}-p_{0}^{2}\right)\left(11 m_{\rho}^{2}+p_{0}^{2}\right)}{m_{\rho}^{2}} K_{4}-4 K_{6}+\frac{p_{0}^{2}}{4 m_{\rho}^{2}} G_{2}\right\}\right] \\
& \operatorname{Re} \Delta \Pi_{\rho L}^{(d)}\left(p_{0}, \vec{p}=0 ; T\right)=\frac{N}{2} \frac{g^{2}}{\pi^{2}}\left[-2 J_{1}^{2}-\frac{1}{3 m_{\rho}^{2}}\left(I_{4}-J_{1}^{4}\right)\right] \\
& \operatorname{Re} \Delta \Pi_{\rho L}^{(e)}\left(p_{0}, \vec{p}=0 ; T\right)=-\frac{N}{2} \frac{g^{2}}{\pi^{2}} \frac{1}{6} G_{2},
\end{aligned}
$$

where functions $F, G, H, I, J$ and $K$ are defined in Appendix B.

Let us first consider the low temperature region $T \ll m_{\rho}$. The functions $F, H$ and $J$ are suppressed by $e^{-m_{\rho} / T}$, and gives negligible contribution. Noting that in the low energy limit $\left(p_{0} \rightarrow 0\right), G_{2} \rightarrow I_{2}=\left(\pi^{2} / 6\right) T^{2}$, we find that the contribution to the $\rho$-meson propergator at low energy is order $T^{2}$ :

$$
\Delta \Pi\left(p_{0}=0, \vec{p}=0 ; T\right) \approx \frac{N}{2} \frac{g^{2}}{72}\left(a^{2}-1\right) T^{2}
$$

Since $a \simeq 2$, the low energy mass parameter decreases as $T^{2}$. However, on mass-shell of $\rho$ meson, $p_{0} \approx m_{\rho}, G_{2}$ term in (b), (c) and (e) are cancelled and

$$
\Delta \Pi\left(p_{0}=m_{\rho}, \vec{p}=0 ; T\right) \approx \frac{N}{2} \frac{g^{2}}{\pi^{2}} \frac{a^{2}}{12} G_{2}
$$

Moreover, since $G_{2} \approx-\frac{\pi^{4}}{15} \frac{T^{4}}{m_{\rho}^{2}}$, the $\rho$-meson mass increases as $T^{4}$ at low temperature dominated by pion-loop effect. 


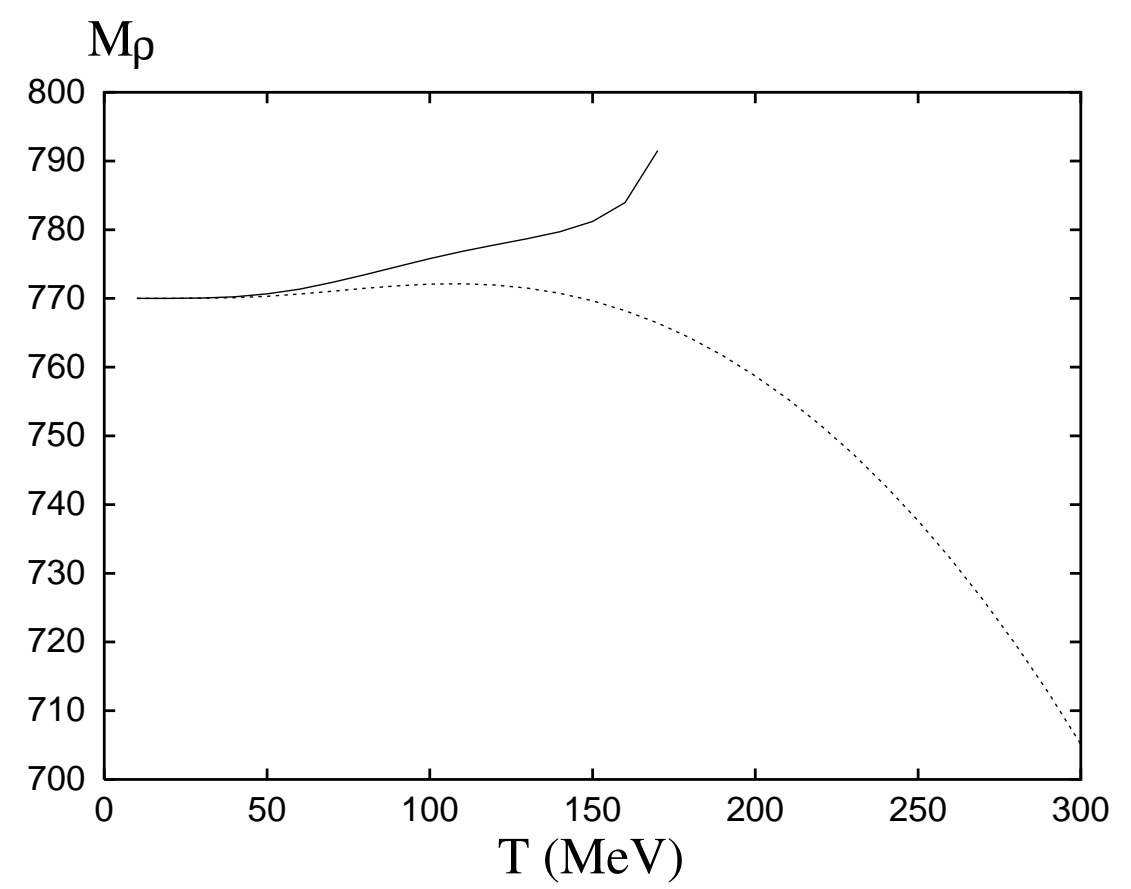

FIG. 6. The temperature dependence of the pole mass of $\rho$ meson. The dotted line denotes the pole mass derived from the pion loop contribution only, while the solid line denotes that from the total contribution.

Next we study the temperature dependence of the pole mass at slightly higher temperature by fully using the expression (25). The pole mass is defined as

$$
M_{\rho}^{2}-m_{\rho}^{2}+\operatorname{Re} \Pi_{\rho L}\left(p_{0}=M_{\rho}, \vec{p}=0 ; T\right)=0 .
$$

We show the temperature dependence of the pole mass by a solid line in Fig. 6. For $T>170$ $\mathrm{MeV}$ there is no solution of Eq. (28) below $1540 \mathrm{MeV}$ when we include all the contributions. Then the solid line terminates at $T \simeq 170 \mathrm{MeV}$. Higher order loop effects may be needed to study such high temperature region. Until $T \simeq 170 \mathrm{MeV}$ the mass of $\rho$ meson increases with temperature. The dotted line denotes the pole mass when we include only the pion loop, where for consistency we replace $z$ in Eq. (24) with

$$
z=-\frac{11}{72} a^{2}
$$

Figure 6 shows that in the high temperature region the pion-loop correction decreases the $\rho$-meson mass, while the $\rho$ loop gives a large positive correction, and as a result the $\rho$-meson mass increases slightly with temperature.

\section{SUMMARY AND DISCUSSION}

In this paper we studied the temperature dependence of the pion decay constant and the $\rho$-meson mass by including the thermal pion and $\rho$-meson effects systematically in the hidden 
local symmetry model. The thermal pion gives a dominant contribution to $f_{\pi}(T)$, and the $\rho$ meson gives a small correction. The low temperature limit of our result is consistent with the chiral perturbation prediction. [23] The inclusion of $\rho$ meson may be a good approximation to $\mathcal{O}\left(p^{4}\right)$ contribution to chiral perturbation analysis.

We also studied the temperature dependence of the parameter $a$, which is related to the successful phenomenological predictions of hidden local symmetry. We showed that the parameter $a$ was stable as the temperature increased, $a(T) \simeq 2$. This may suggest that the phenomenological predictions of hidden local symmetry holds at finite temperature in good approximation. Our result shows that the parameter $a$ does not change at all if we take $a=1$ from the beginning. This implies that in the "vector limit" [26] the thermal effects of the $\rho$ meson do not induce deviation from $a=1$.

We calculated the temperature dependence of the mass of $\rho$ meson. We showed that the low-energy mass parameter decreased as $T^{2}$ in the low temperature region. However, the $\rho$-meson pole mass increases as $T^{4}$ dominated by pion-loop effect, which is consistent with the result by the current algebra analysis. [5] In the high temperature region pion gives a negative contribution, while the $\rho$ loop gives a large positive correction, and the $\rho$-meson mass increases with temperature. The correction from pion loop in our model does not seem to agree with previous analysis [6] at slightly higher temperature. The difference between the result of our model and that of previous models comes from the fact that there is no pion tad-pole contribution at one loop in our model. The tad-pole diagram gives a relatively large positive contribution to the $\rho$-meson mass in the previous models. Instead in our model, $\rho$ meson gives a positive contribution, and it overcomes the pion-loop contribution. Although each correction to the $\rho$-meson mass is small, it is interesting to point out that the $\rho$-loop contribution is bigger than the pion-loop contribution for $T \geq 50 \mathrm{MeV}$. Our analysis implies that the inclusion of $\rho$ meson itself is important to study the temperature dependence of $\rho$-meson mass near critical temperature.

In the high temperature region one-loop approximation might be too crude to study the temperature dependences. Although we can expect that the qualitative structure does not change, it seems to be interesting to include the higher order effect, for example, by using the temperature dependent renormalization group analysis [27].

Finally we make a comment on the correction to the $\rho$-meson mass from the $\omega$ - $\pi$ loop. In this paper we did not include the anomalous interaction terms such as $\omega-\rho-\pi$, since they are $\mathcal{O}\left(p^{4}\right)$ terms in a chiral counting rule [17] and expected to give higher order effects. The $\omega-\pi$ loop correction to the $\rho$-meson self-energy is $\mathcal{O}\left(p^{8}\right)$, while the $\rho$-meson loop generates $\mathcal{O}\left(p^{4}\right)$ correction. Apparently the $\omega-\pi$ loop does not generate any correction to the low-energy mass parameter. To the pole mass, there is a suppression factor $(g / 4 \pi)^{4}$ for the $\omega-\pi$ loop correction compared with the $\rho$-loop correction, then we expect that the correction is small. 


\section{ACKNOWLEDGMENT}

We would like to thank to Prof. Koichi Yamawaki for suggesting this subject and for stimulating discussions. We would like to thank also to people in Nagoya University where the final stage of this work was done. We are also grateful to Prof. Joe Schechter for useful comment. 


\section{APPENDICES}

\section{APPENDIX A: POLARIZATION TENSORS}

At finite temperature, the polarization tensor is no longer restricted to be Lorentz covariant, but only $O(3)$ covariant. Then the polarization tensors can be expressed by four independent symmetric $O(3)$ tensors. Here we list up the polarization tensors at finite temperature: 28,29]

$$
\begin{aligned}
P_{T \mu \nu} & =g_{\mu i}\left(\delta_{i j}-\frac{\vec{p}_{i} \vec{p}_{j}}{|\vec{p}|^{2}}\right) g_{j \nu} \\
& =\left\{\begin{array}{l}
P_{T 00}=P_{T 0 i}=P_{T i 0}=0 \\
P_{T i j}=\delta_{i j}-\frac{\vec{p}_{i} \vec{p}_{j}}{|\vec{p}|^{2}},
\end{array}\right. \\
P_{L \mu \nu} & \equiv-\left(g_{\mu \nu}-\frac{p_{\mu} p_{\nu}}{p^{2}}\right)-P_{T \mu \nu} \\
& =\left(g_{\mu 0}-\frac{p_{\mu} p_{0}}{p^{2}}\right) \frac{p^{2}}{|\vec{p}|^{2}}\left(g_{0 \nu}-\frac{p_{0} p_{\nu}}{p^{2}}\right), \\
P_{C \mu \nu} & \equiv \frac{1}{\sqrt{2}|\vec{p}|}\left[\left(g_{\mu 0}-\frac{p_{\mu} p_{0}}{p^{2}}\right) p_{\nu}+p_{\mu}\left(g_{0 \nu}-\frac{p_{0} p_{\nu}}{p^{2}}\right)\right], \\
P_{D \mu \nu} & \equiv \frac{p_{\mu} p_{\nu}}{p^{2}},
\end{aligned}
$$

where $p^{\mu}=\left(p_{0}, \vec{p}\right)$ is four-momentum.

The following formulae are convenient:

$$
\begin{aligned}
P_{L \mu \alpha} P_{L}^{\alpha \nu} & =-P_{L \mu}{ }^{\nu}, \\
P_{T \mu \alpha} P_{T}^{\alpha \nu} & =-P_{T \mu}{ }^{\nu}, \\
P_{C \mu \alpha} P_{C}^{\alpha \nu} & =\frac{1}{2}\left(P_{L \mu}{ }^{\nu}+P_{D \mu}{ }^{\nu}\right) \\
P_{D \mu \alpha} P_{D}^{\alpha \nu} & =P_{D \mu}{ }^{\nu}, \\
P_{L \mu \alpha} P_{T}^{\alpha \nu} & =P_{C \mu \alpha} P_{T}^{\alpha \nu}=P_{D \mu \alpha} P_{T}^{\alpha \nu}=P_{D \mu \alpha} P_{L}^{\alpha \nu}=0 \\
P_{C \mu \alpha} P_{L}^{\alpha \nu} & =-P_{D \mu \alpha} P_{C}^{\alpha \nu}=-\frac{p_{\mu}}{\sqrt{2}|\vec{p}|}\left(g_{0}^{\nu}-\frac{p_{0} p^{\nu}}{p^{2}}\right) .
\end{aligned}
$$

For the transversal tensor $p_{\mu} \Pi^{\mu \nu}(p)=0$ we can decompose it into

$$
\Pi_{\mu \nu}(p)=P_{L \mu \nu} \Pi_{L}(p)+P_{T \mu \nu} \Pi_{T}(p),
$$

where $\Pi_{L}$ and $\Pi_{T}$ are given by

$$
\begin{aligned}
& \Pi_{L}=\frac{p^{2}}{|\vec{p}|^{2}} \Pi_{00}, \\
& \Pi_{T}=-\frac{1}{2}\left\{\Pi_{j}^{j}+\frac{p_{0}^{2}}{|\vec{p}|^{2}} \Pi_{00}\right\} .
\end{aligned}
$$




\section{APPENDIX B: FUNCTIONS}

Here we list the functions used in this paper.

Functions used in the expressions of $f_{\pi}$ and $f_{\sigma}$ in Eqs. (14) and (18) are defined as follows;

$$
\begin{gathered}
I_{n}(T) \equiv \int_{0}^{\infty} d \mathrm{k} \frac{\mathrm{k}^{n-1}}{e^{\mathrm{k} / T}-1}=\widetilde{I}_{n} T^{n}, \\
\widetilde{I}_{n}=\int_{0}^{\infty} d y \frac{y^{n-1}}{e^{y}-1}=(n-1) ! \zeta(n), \\
\widetilde{I}_{2}=\frac{\pi^{2}}{6}, \quad \widetilde{I}_{4}=\frac{\pi^{4}}{15}, \quad \widetilde{I}_{6}=\frac{8 \pi^{6}}{63}, \\
J_{m}^{n}\left(m_{\rho} ; T\right) \equiv \int_{0}^{\infty} d \mathrm{k} \frac{1}{e^{\omega / T}-1} \frac{\mathrm{k}^{n}}{\omega^{m}} \quad ; \quad n, m: \text { integer }, \\
\omega \equiv \sqrt{\mathrm{k}^{2}+m_{\rho}^{2}} .
\end{gathered}
$$

We also define the functions in the $\rho$-meson propagator as follows:

$$
\begin{aligned}
F_{3}^{n}\left(p_{0} ; m_{\rho} ; T\right) & \equiv \int_{0}^{\infty} d \mathrm{k} \mathcal{P} \frac{1}{e^{\omega / T}-1} \frac{4 \mathrm{k}^{n}}{\omega\left(4 \omega^{2}-p_{0}^{2}\right)} \\
G_{n}\left(p_{0} ; T\right) & \equiv \int_{0}^{\infty} d \mathrm{k} \mathcal{P} \frac{\mathrm{k}^{n-1}}{e^{\mathrm{k} / T}-1} \frac{4 \mathrm{k}^{2}}{4 \mathrm{k}^{2}-p_{0}^{2}} \\
& =I_{n}(T)+\int_{0}^{\infty} d \mathrm{k} \mathcal{P} \frac{\mathrm{k}^{n-1}}{e^{\mathrm{k} / T}-1} \frac{p_{0}^{2}}{4 \mathrm{k}^{2}-p_{0}^{2}} \\
H_{1}^{n}\left(p_{0} ; m_{\rho} ; T\right) & \equiv \int_{0}^{\infty} d \mathrm{k} \mathcal{P} \frac{1}{e^{\omega / T}-1} \frac{\mathrm{k}^{n}}{\omega} \frac{1}{\left(m_{\rho}^{2}-p_{0}^{2}\right)^{2}-4 \mathrm{k}^{2} p_{0}^{2}} \\
K_{n}\left(p_{0} ; m_{\rho} ; T\right) & \equiv \int_{0}^{\infty} d \mathrm{k} \mathcal{P} \frac{\mathrm{k}^{n-1}}{e^{\mathrm{k} / T}-1} \frac{1}{\left(m_{\rho}^{2}-p_{0}^{2}\right)^{2}-4 \mathrm{k}^{2} p_{0}^{2}},
\end{aligned}
$$

where $\mathcal{P}$ denotes the principal part.

\section{APPENDIX C: $\rho$ AND $\sigma$ PROPAGATORS}

We introduced an $R_{\xi}$-like gauge-fixing term (8) for eliminating the tree-level $\rho$ - $\sigma$ mixing existing in the Lagrangian (4). Generally, a new $\rho-\sigma$ mixing is generated by one-loop effects. At zero temperature one-loop corrections do not generate any corrections to the $\rho$ - $\sigma$ mixing when we use the Landau gauge. Here we calculate the $\rho-\sigma$ mixing at finite temperature. There are three diagrams contributing to the $\rho-\sigma$ mixing at one loop in the Landau gauge: (a) $\pi$-loop; (b) $\pi$ tad-pole; (c) $\sigma$ tad-pole, which are shown in Fig. 7. These diagrams give corrections to the $\rho-\sigma$ mixing given by

$$
F_{\mu}\left(p_{0}, \vec{p} ; T\right)=\left[\left(N(a-1)+\frac{1}{N}\right) \frac{(a+1) g}{8 \pi^{2} f_{\sigma}} I_{2}\right] p_{\mu}
$$


where $I_{2}$ is defined in Eq. (B1). It is remarkable to see that all the contributions are proportional to four-momentum $p_{\mu}$.

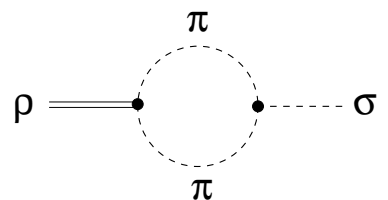

(a)

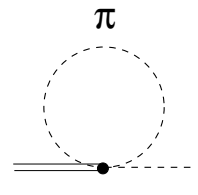

(b)

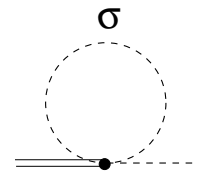

(c)

FIG. 7. Diagrams contributing to the $\rho-\sigma$ mixing at one loop in the Landau gauge.

When $\rho-\sigma$ mixing is proportional to four-momentum, the inverse-propagator matrix for $\rho$ and $\sigma$, in general, takes the form given by

$$
\Delta_{(\mu, \nu)}^{-1}=\left(\begin{array}{cc}
\widetilde{D}_{\sigma}^{-1} & i F p_{\nu} \\
-i F p_{\mu} & \widetilde{D}_{\mu \nu}^{-1}
\end{array}\right)
$$

Here $\widetilde{D}_{\sigma}^{-1}$ and $\widetilde{D}_{\mu \nu}^{-1}$ are inverse $\sigma$ and $\rho$ propagators before diagonalization. The inverse $\rho$ propagator $\widetilde{D}_{\mu \nu}^{-1}$ is projected to four polarizations by the polarization tensor given in Eq. (A1):

$$
\widetilde{D}_{\mu \nu}^{-1} \equiv P_{T \mu \nu} \widetilde{\Pi}_{T}+P_{L \mu \nu} \widetilde{\Pi}_{L}+P_{C \mu \nu} \widetilde{\Pi}_{C}+P_{D \mu \nu} \widetilde{\Pi}_{D}
$$

After some calculations we obtain the $\rho$ - $\sigma$ propagator matrix:

$$
\Delta_{(\mu, \nu)}^{-1}=\left(\begin{array}{cc}
A & i p_{0} B i p_{j} C \\
-i p_{0} B & D_{\mu \nu} \\
-i p_{i} C &
\end{array}\right)
$$

where

$$
\begin{aligned}
A & =\frac{\widetilde{D}_{\sigma}\left[\widetilde{\Pi}_{L} \widetilde{\Pi}_{D}-\left(\widetilde{\Pi}_{C}\right)^{2} / 2\right]}{\widetilde{\Pi}_{L} \widetilde{\Pi}_{D}-\left(\widetilde{\Pi}_{C}\right)^{2} / 2-p^{2} F^{2} \widetilde{D}_{\sigma} \widetilde{\Pi}_{L}}, \\
B & =\frac{\widetilde{D}_{\sigma} F\left[\bar{p} \widetilde{\Pi}_{C} / \sqrt{2}-p_{0} \widetilde{\Pi}_{L}\right]}{\bar{p}\left[\widetilde{\Pi}_{L} \widetilde{\Pi}_{D}-\left(\widetilde{\Pi}_{C}\right)^{2} / 2-p^{2} F^{2} \widetilde{D}_{\sigma} \widetilde{\Pi}_{L}\right]}, \\
C & =\frac{\widetilde{D}_{\sigma} F\left[p_{0} \widetilde{\Pi}_{C} / \sqrt{2}-\bar{p} \widetilde{\Pi}_{L}\right]}{p_{0}\left[\widetilde{\Pi}_{L} \widetilde{\Pi}_{D}-\left(\widetilde{\Pi}_{C}\right)^{2} / 2-p^{2} F^{2} \widetilde{D}_{\sigma} \widetilde{\Pi}_{L}\right]},
\end{aligned}
$$

and

$$
D_{\mu \nu}=\frac{P_{T \mu \nu}}{\widetilde{\Pi}_{T}}+\frac{P_{L \mu \nu}\left[\widetilde{\Pi}_{D}-p^{2} \widetilde{D}_{\sigma} F^{2}\right]+P_{C \mu \nu} \widetilde{\Pi}_{C}+P_{D \mu \nu} \widetilde{\Pi}_{L}}{\widetilde{\Pi}_{L} \widetilde{\Pi}_{D}-\left(\widetilde{\Pi}_{C}\right)^{2} / 2-p^{2} F^{2} \widetilde{D}_{\sigma} \widetilde{\Pi}_{L}} .
$$


In the $R_{\xi}$ gauge, the inverse free propagator of $\rho$ meson is give by

$$
D_{0 \nu}^{-1}=-\left(P_{L \mu \nu}+P_{T \mu \nu}\right)\left(p^{2}-m_{\rho}^{2}\right)+\frac{p^{2}-\alpha m_{\rho}^{2}}{\alpha} \frac{p_{\mu} p_{\nu}}{p^{2}}
$$

where $\alpha$ is the gauge parameter. The inverse full propagator is defined by $D_{\mu \nu}^{-1}=D_{0}^{-1}{ }_{\mu \nu}-$ $\Pi_{\mu \nu}$, where the $\rho$ meson self-energy $\Pi_{\rho}^{\mu \nu}$ is expanded by four independent polarization tensors listed in Eq. (A1):

$$
\Pi_{\rho}^{\mu \nu}=P_{T}^{\mu \nu} \Pi_{\rho T}+P_{L}^{\mu \nu} \Pi_{\rho L}+P_{C}^{\mu \nu} \Pi_{\rho C}+P_{D}^{\mu \nu} \Pi_{\rho D}
$$

By using these quantities, four components of the inverse $\rho$ propagator in Eq. (C3) are given by

$$
\begin{aligned}
\widetilde{\Pi}_{T} & =-\left(p^{2}-m_{\rho}^{2}+\Pi_{\rho T}\right), \\
\widetilde{\Pi}_{L} & =-\left(p^{2}-m_{\rho}^{2}+\Pi_{\rho L}\right), \\
\widetilde{\Pi}_{C} & =-\Pi_{\rho C}, \\
\widetilde{\Pi}_{D} & =\frac{p^{2}-\alpha m_{\rho}^{2}}{\alpha}-\Pi_{\rho D} .
\end{aligned}
$$

If we take the Landau gauge $\alpha=0$, the $\rho$ - $\sigma$ propagator matrix reduces to a simple form:

$$
\begin{aligned}
& A=\widetilde{D}_{\sigma}, \quad B=C=0, \\
& D_{\mu \nu}=-\frac{P_{T \mu \nu}}{p^{2}-m_{\rho}^{2}+\Pi_{\rho T}}-\frac{P_{L \mu \nu}}{p^{2}-m_{\rho}^{2}+\Pi_{\rho L}} .
\end{aligned}
$$


[1] R.D. Pisarski, BNL-Preprint hep-ph/9503330, (unpublished).

[2] G.E. Brown and M. Rho, Phys. Rept. 269, (1996).

[3] T. Hatsuda, H. Shiomi and H. Kuwabara, Prog.Theor.Phys. 95, 1009 (1996).

[4] G.E. Brown and M. Rho, Phys. Rev. Lett. 66, 2720 (1991).

[5] M. Dey, V.L. Eletsky and B.L. Ioffe, Phys. Lett. B 252, 620 (1990).

[6] R.D. Pisarski, Phys. Rev. D 52, 3773 (1995);

C. Song, Phys. Rev. D 53, 3962 (1996);

S.H. Lee, C. Song and H. Yabu, Phys. Lett. B 341, 407 (1995);

C. Gale and J. Kapusta, Nucl. Phys. B357, 65 (1991).

[7] H. Shiomi and T. Hatsuda, Phys, Lett. B 334, 281 (1994).

[8] P. Gerber and H. Leutwyler, Nucl. Phys. B321, 387 (1989).

[9] M. Bando, T. Kugo and K. Yamawaki, Phys. Rep. 164, 217 (1988).

[10] M. Bando, T. Kugo, S. Uehara, K. Yamawaki and T. Yanagida, Phys. Rev. Lett. 54, 1215 (1985).

[11] J.J. Sakurai, Currents and Mesons (Univ. Chicago Press, Chicago, 1969).

[12] K. Kawarabayashi and M. Suzuki, Phys. Rev. Lett. 16, 255 (1966);

Riazuddin and Fayyazuddin, Phys. Rev. 147, 1071 (1966).

[13] M. Bando, T. Kugo and K. Yamawaki, Nucl. Phys. B259, 493 (1985).

[14] M. Bando, T. Kugo and K. Yamawaki, Prog. Theor. Phys. 73, 1541 (1985).

[15] M. Harada, T. Kugo and K. Yamawaki, Phys. Rev. Lett. 71, 1299 (1993); Prog. Theor. Phys. 91, 801 (1994).

[16] M. Harada and K. Yamawaki, Phys. Lett. B 297, 151 (1992).

[17] M. Tanabashi, Phys. Lett. B 316, 534 (1993).

[18] T. Matsubara, Prog. Theor. Phys. 14, 351 (1955); see also Ref. 28].

[19] Ö. Kaymakcalan and J. Schechter, Phys. Rev. D 31, 1109 (1985).

[20] M. Bando, T. Fujiwara and K. Yamawaki, Prog. Theor. Phys. 79, 1140 (1988); 
K. Yamawaki, in Proc. 1985 INS Symp. on Composite models of quarks and leptons (Tokyo, August, 1985), edited by H. Terazawa and M. Yasuè (INS, University of Tokyo, Tokyo, 1985).

[21] K. Fujikawa, B.W. Lee and A.I. Sanda, Phys. Rev. D 6, 2923 (1972).

[22] A. Bochkarev and J. Kapusta, Phys. Rev. D 54, 4066(1996).

[23] J. Gasser and H. Leutwyler, Phys. Lett. B 184, 83 (1987).

[24] J. Gasser and H. Leutwyler, Ann. Phys. (N.Y.) 158, 142 (1984).

[25] G. Ecker, J. Gasser, A. Pich, and E. de Rafael, Nucl. Phys. B321, 311 (1989);

G. Ecker, J. Gasser, H. Leutwyler, A. Pich and E. de Rafael, Phys. Lett. B 233, 425 (1989);

J.F. Donoghue, C. Ramirez and G. Valencia, Phys. Rev. D 39, 1947 (1989).

[26] H. Georgi, Phys. Rev. Lett. 63, 1917 (1989); Nucl. Phys. B 331, 311 (1990).

[27] H. Matsumoto, Y. Nakano and H. Umezawa, Phys. Rev. D 29, 1116 (1984).

[28] T. Toimele, Int. J. Theor. Phys. 24, 901 (1985), and references therin.

[29] N. Doray and N.E. Mavromatos, Nucl. Phys. B, 386, 614 (1992). 